
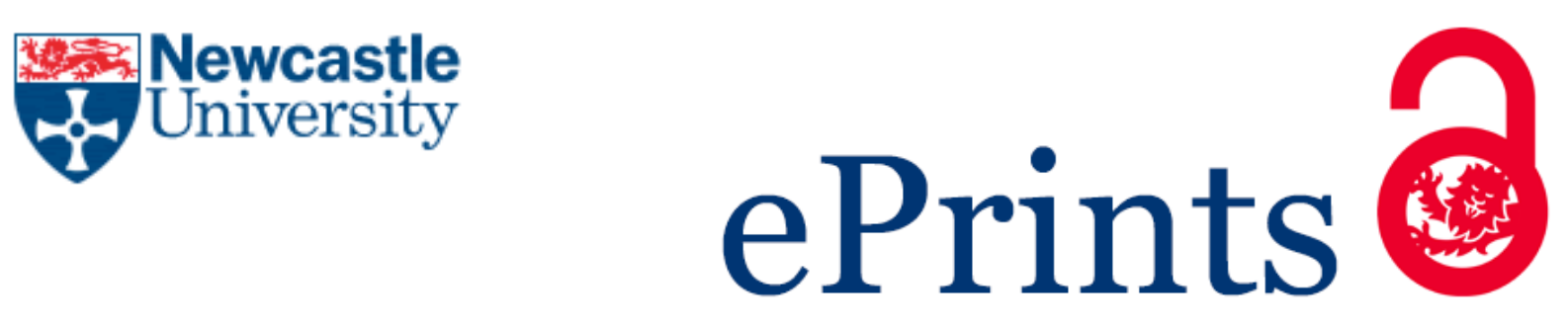

Rockley K, Gill JH.

Characterisation of Novel Molecular Mechanisms Involved in AnthracyclineInduced Cardiotoxicity

Journal of Pharmacological and Toxicological Methods 2017, 88(Part 2), 202

\title{
Copyright:
}

(C) 2017. This manuscript version is made available under the CC-BY-NC-ND 4.0 license

DOI link to article:

https://doi.org/10.1016/j.vascn.2017.09.112

Date deposited:

$07 / 03 / 2018$

Embargo release date:

13 November 2018

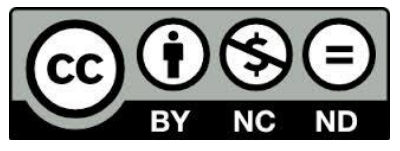

This work is licensed under a

Creative Commons Attribution-NonCommercial-NoDerivatives 4.0 International licence 


\section{Characterisation of Novel Molecular Mechanisms Involved in Anthracycline- Induced Cardiotoxicity}

Recent advances in cancer treatment have improved cancer survivorship; however as a result the incidence of cancer therapy induced cardiotoxicity has increased. This major complication can present both as an acute toxicity or a chronic toxicity and can impose substantial detriment to both the quality of life and survival of patients. Consequently, greater understanding of the molecular mechanisms responsible for these toxicities, and identification of therapeutic strategies to mitigate and overcome these toxicities are significantly important. Recently, clinical studies have demonstrated that administration of drugs that act upon the angiotensin system may reduce the cardiotoxicity of anthracyclines. However, despite showing promise, the molecular mechanisms responsible for toxicity mitigation are currently unknown. This study has utilised a variety of human cardiomyocyte in vitro models and real-time impedance-based cell analyses (xCELLigence technology) to demonstrate induction of cardiomyocyte hypertrophy by doxorubicin, and through its reduction by blockade of the angiotensin pathway, implicates synergism between doxorubicinmediated toxicity and angiotensin signalling in cardiomyocytes. Beat characteristics of iPSC derived cardiomyocytes have also been assessed using XCELLigence Cardio technology during exposure to doxorubicin alone and during angiotensin blockade in an attempt to elucidate the effects of cardiomyocyte hypertrophy on contractility of these cells. The methodologies used in this study offer a robust clinically relevant model for assessing drug-induced cardiotoxicity, with the study identifying a novel mechanism for anthracycline-induced cardiotoxicity. 